\title{
Paracoccidioidomicose da região ocular: relato de dois casos e revisão da literatura*
}

\section{Ocular paracoccidioidomycosis: report of two cases and review of literature*}

\author{
Roberto Lopes Gervini ${ }^{1}$ \\ Gerson Vettorato ${ }^{2}$
}

\author{
Sergio Martinez Lecompte ${ }^{3}$ \\ Tatiana Basso Biasi ${ }^{3}$
}

\author{
Fernanda Goulart Ruthner ${ }^{3}$ \\ Fernando Leite Kronbauer ${ }^{4}$
}

\begin{abstract}
Resumo: No Brasil, a paracoccidioidomicose é doença endêmica que pode afetar diversos órgãos, sendo pouco comum o envolvimento da região ocular. Nessa forma, é freqüente o acometimento de conjuntivas e pálpebras, configurando-se em afecção importante a considerar no diagnóstico diferencial das lesões da região orbitária. Mesmo nas formas localizadas deve ser feita a avaliação sistêmica, uma vez que a ocorrência da paracoccidioidomicose na região ocular pode ser parte de doença multifocal.

Relatam-se dois casos de paracoccidioidomicose da região ocular e faz-se uma revisão dos 55 casos descritos na literatura até o momento.

Palavras-chave: conjuntiva; doenças palpebrais; epidemiologia; paracoccidioidomicose
\end{abstract}

\begin{abstract}
Summary: Paracoccidioidomycosis is an endemic disease in Brazil that commonly affects multiple organs, although ocular involvement is rare. The ocular form of this disease affects predominantly the eyelids and conjunctiva, bighlighting the importance for dermatologists to consider this disease in the differential diagnosis for lesions within the orbital area. Even with localized forms of the disease, a complete systemic examination should be done bearing in mind that the exclusive ocular form of paracoccidioidomycosis can frequently bide a multi-systemic disease.

We describe two cases of the ocular form of paracoccidioidomycosis with a revision of 55 cases described in the literature.
\end{abstract}

Key-words: conjunctiva; eyelid diseases; epidemiology; paracoccidioidomycosis.

\section{INTRODUÇÃO}

A paracoccidioidomicose é micose sistêmica causada pelo fungo dimórfico Paracoccidioides brasiliensis, originalmente descrita por Adolfo Lutz, no Brasil, em 1908. ${ }^{1,2}$ A doença ocorre sobretudo em adultos entre 30 e 60 anos de idade, sendo rara em crianças e adultos jovens..$^{2-5}$ É mais freqüente no sexo masculino, com a proporção homem/mulher nas áreas de endemicidade de 13:1.,.$^{3,6,7}$

\section{INTRODUCTION}

Paracoccidioidomycosis is systemic mycosis caused by the dimorphic fungi Paracoccidioides brasiliensis, originally described by Adolfo Lutz (Brazil) in 1908.,2 The disease occurs above all in adults from 30 to 60 years of age and is rare in children and young adults. ${ }^{2-5}$ It is more frequent in the male sex, with a male/female ratio in endemic areas of 13:1.,6,7

\footnotetext{
Recebido em 13.08.2001. / Received in August, $13^{\text {th }}$ of 2001.

Aprovado pelo Conselho Consultivo e aceito para publicação em 24.05.2002. / Approved by the Consultive Council and accepted for publication in May, $24^{\text {th }}$ of 2002.

* Trabalho realizado no Complexo Hospitalar Santa Casa de Porto Alegre, Serviço de Dermatologia/UFRGS. / Work done at "Complexo Hospitalar Santa Casa de Porto Alegre, Serviço de Dermatologia/UFRGS".

Chefe do Serviço de Dermatologia/UFRGS. Complexo Hospitalar Santa Casa. Porto Alegre, Rio Grande do Sul, Brasil. / Head of Dermatology Service /UFRGS. Complexo Hospitalar Santa Casa. Porto Alegre, Rio Grande do Sul, Brazil.

Médico Dermatologista responsável pelo setor de Micologia. Serviço de Dermatologia/UFRGS. Complexo Hospitalar Santa Casa. Porto Alegre, Rio Grande do Sul, Brasil. / M.D. Dermatologist, responsible for the Mycology sector, Dermatology Service/UFRGS. Complexo Hospitalar Santa Casa. Porto Alegre, Rio Grande do Sul, Brazil

Médicos Residentes do Serviço de Dermatologia/UFRGS. Complexo Hospitalar Santa Casa. Porto Alegre, Rio Grande do Sul, Brasil. / Residents M.D., Dermatology Service /UFRGS. Complexo Hospitalar Santa Casa. Porto Alegre, Rio Grande do Sul, Brazil.

${ }^{4}$ Médico Residente do Serviço de Oftalmologia. Complexo Hospitalar Santa Casa. Porto Alegre, Rio Grande do Sul, Brasil. / Resident M.D., Ophthalmology Service. Complexo Hospitalar

Santa Casa. Porto Alegre, Rio Grande do Sul, Brazil.

○2004 by Anais Brasileiros de Dermatologia
} 
Na maioria dos casos (entre 70 e $80 \%$ ) a paracoccidioidomicose é doença multifocal. ${ }^{4,5}$ No entanto, quando unifocal, o acometimento pulmonar é o mais comum, sendo pouco freqüente o envolvimento apenas mucocutâneo ou de outros focos. ${ }^{8}$ As localizações extrapulmonares ocorrem em geral na pele do rosto e nas mucosas oral, faríngea, laríngea e nasal. ${ }^{2,9}$ Raramente pode haver envolvimento de outras mucosas, como a anal, a genital ou a ocular. ${ }^{10} \mathrm{O}$ acometimento dos linfonodos também é freqüente, sendo os cervicais e supraclaviculares mais comumente afetados. .,4,11 $^{2,11}$

Neste trabalho, são relatados dois casos da doença com localização ocular. Também é realizada uma revisão dos casos de paracoccidioidomicose com envolvimento ocular descritos na literatura.

\section{RELATO DOS CASOS}

\section{Caso 1}

Paciente do sexo masculino, de 67 anos, agricultor, procedente de Encantado, Rio Grande do Sul. Relatou o surgimento de pequenas lesões ulceradas, com seis meses de evolução, localizadas na pálpebra inferior direita e antebraço esquerdo, ambas de crescimento lento. Apresentava história pregressa de tabagismo e alcoolismo.

O exame dermatológico mostrou, na pálpebra inferior direita, pequena lesão ulcerada, rasa, com base infiltrada, esboçando discreta vegetação e pontilhado hemorrágico na superfície (Figura 1). No antebraço esquerdo, presença de pequenas lesões ulceradas, com centro coberto por crosta hemorrágica e exsudato fibrinoso. Ao exame oftalmológico observou-se envolvimento pálpebro-conjuntival, com conseqüente lagoftalmo temporal direito e ceratite de exposição inferior. Exame do globo ocular sem anormalidades. No exame micológico direto da lesão cutânea observou-se células arredondadas, birrefringentes, com vários brotamentos compatíveis com Paracoccidiodes brasiliensis (Figura 2). A cultura confirmou o diagnóstico. O exame histopatológico da lesão palpebral evidenciou infiltrado inflamatório granulomatoso com a presença do fungo. $\mathrm{Na}$ radiografia de tórax não foram encontradas alterações. A sorologia pela técnica de imunodifusão dupla em ágar-gel resultou positiva para paracoccidioidomicose. Outros exames laboratoriais, como hemograma, provas de

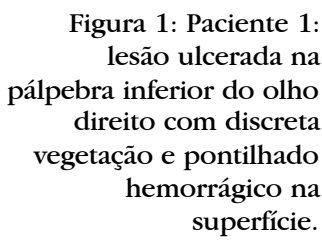

In most cases (between 70 and 80\%) paracoccidioidomycosis is a multifocal disease. ${ }^{4,5}$ However, when unifocal, lung involvement is predominant and mucocutaneous involvement or other foci are somewhat infrequent. ${ }^{8}$ Extrapulmonary locations occur in general in the skin of the face and in the oral, pharyngeal, laryngeal and nasal mucous membranes. ${ }^{2,9}$ There is rarely involvement of other mucous membranes, such as the anal, genital or occular membranes..$^{10}$ Involvement of the lymph nodes is also frequent and the cervical and supraclavicular lymph nodes are the most commonly affected. ${ }^{2,411}$

This work presents two cases reports of the disease with ocular location and also a revision of the cases of paracoccidioidomycosis with ocular involvement described in the literature.

\section{CASE REPORTS \\ Case 1}

Patient male, 67 years old, farmer, resident in Encantado, Rio Grande do Sul. He complained of the appearance of small ulcerated lesions, with six months of evolution, located in the right inferior eyelid and left forearm, both of slow growth. He presented a prior history of smoking and alcoholism.

Dermatological exam showed in the right inferior eyelid a small, shallow ulcerated lesion with infiltrated base, discreet vegetation and stippled hemorrhage in the surface (Figure 1). In the left forearm there were small ulcerated lesions, with center covered by hemorrhagic crust and fibrinous exudate. Ophthalmologic exam revealed palpebral-conjunctival involvement, with consequent right temporal lagophthalmic and keratitis of inferior exposure. Examination of the eyeball showed no abnormalities. Direct mycological exam of the cutaneous lesion detected round, birefringent cells with several branches compatible with Paracoccidiodes brasiliensis (Figure 2). Culture confirmed the diagnosis. Histopathologic exam of the palpebral lesion revealed a granulomatous inflammatory infiltrate with the presence of fungi. No alterations were found in the chest $x$-ray. Serology using the double immunodiffusion in agargel technique resulted positive for paracoccidioidomycosis. Other laboratorial exams, such as blood count

Figure 1: Patient 1:

Ulcerated lesion in the lower eyelid of the right eye with discrete vegetation and stippled bemorrbage in the surface. 
função hepática e renal, não apresentaram alterações. O tratamento foi realizado com itraconazol $200 \mathrm{mg} / \mathrm{dia}$ durante três meses, com diminuição posterior da dose para $100 \mathrm{mg} /$ dia até o término do sexto mês de tratamento, tendo ocorrido o desaparecimento das lesões mucosas e cutâneas.

\section{Caso 2}

Paciente de 46 anos, do sexo masculino, procedente de zona urbana (Esteio, RS), com surgimento, há quatro meses, de lesões papulosas assintomáticas na pálpebra superior direita. Ao exame dermatológico apresentava lesão ulcerada com fundo discretamente vegetante, pontos hemorrágicos e bordas infiltradas, arredondadas e translúcidas, nas pálpebras superior e inferior direita. (Figura 3). Ao exame oftalmológico observou-se comprometimento da margem palpebral e conjuntival superior, com conseqüente ceratite de exposição inferior e madarose ciliar. $\mathrm{O}$ exame micológico direto mostrou numerosas células arredondadas com brotamentos múltiplos. A cultura em meio Sabouraud, após 30 dias, foi negativa. O exame histopatológico da lesão cutânea demonstrou infiltrado inflamatório granulomatoso com a presença do Paracoccidioides brasiliensis. Os exames laboratoriais (hemograma, eletrólitos, função renal, hepática, glicemia de jejum, anti-HIV e VDRL) estavam normais. A radiografia de tórax mostrou opacidades presumivelmente consolidativas nos lobos médio e inferior direitos e provável hiperinsuflação pulmonar, sugestivas de doença broncopulmonar obstrutiva crônica. O exame micológico direto do escarro e o cultivo a 25 graus demonstraram-se negativos. Foi instituído tratamento com itraconazol $200 \mathrm{mg} /$ dia durante um mês, posteriormente reduzindo-se a dose para $100 \mathrm{mg} / \mathrm{dia}$, a qual foi mantida durante cinco meses. Inicialmente associou-se prednisona $60 \mathrm{mg} / \mathrm{dia}$, sus-

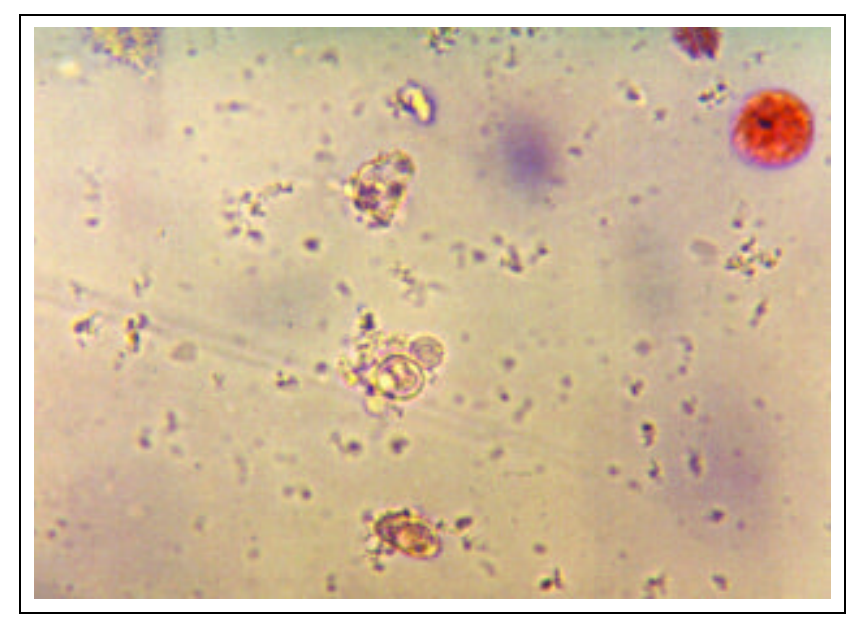

Figura 2: Paciente 1: exame micológico direto da lesão cutânea evidenciando células arredondadas, birrefringentes, com brotamentos compatíveis com Paracoccidiodes brasiliensis. / Figure 2: Patient 1: Direct mycological exam of cutaneous lesion, showing rounded, birefringent cells and branches compatible with Paracoccidioides brasiliensis. and tests of hepatic and renal function, did not present any alterations. Treatment was initiated with itraconazole, 200mg/day for three months and subsequent decrease of the dose to $100 \mathrm{mg} /$ day at the end of the sixth month of treatment, with disappearance of the mucous and cutaneous lesions.

\section{Case 2}

Patient, 46 years old, male, resident in an urban area (Esteio, RS), with appearance four months earlier of asymptomatic papular lesions in the right superior eyelid. At dermatological exam he presented ulcerated lesion with discreetly vegetative bed, hemorrhagic points and infiltrated, round and translucent borders, in the superior and inferior right eyelids (Figure 3). Ophthalmologic exam revealed involvement of the palpebral and superior conjunctival margin, with consequent inferior exposure keratitis and ciliary madarosis. Direct mycological exam showed numerous round cells with multiple branches. Culture in Sabouraud's agar, after 30 days, was negative. Histopathologic exam of the cutaneous lesion demonstrated inflammatory granulomatous infiltrate and the presence of Paracoccidioides brasiliensis. Laboratory exams (blood count, electrolytes, renal and hepatic function, fast glycemia, anti-HIV and VDRL test) were all normal. Chest $x$-ray showed opacities presumably consolidant in the right median and inferior lobes and probable lung hyperinflation, suggestive of chronic bronchopulmonary obstructive disease. Direct mycological exam of the spittle and cultivation at $25^{\circ} \mathrm{C}$ were negative. Treatment was instituted with itraconazole at 200mg/day for one month, later being reduced to $100 \mathrm{mg} /$ day, which was maintained for five months. Initially this was associated with prednisone at 60mg/day, which was suspended after 15 days fol-

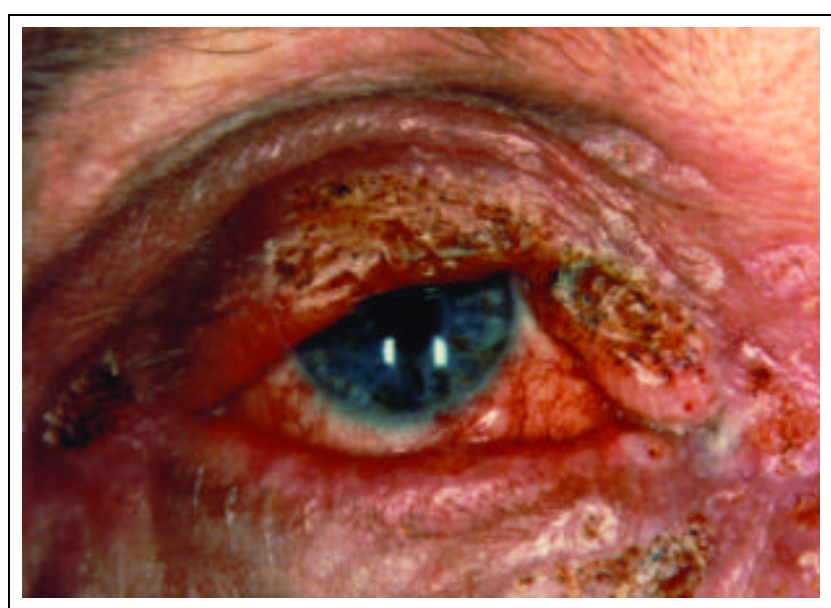

Figura 3: Paciente 2: lesão ulcerovegetante com fundo evidenciando pontilhado hemorrágico e bordas discretamente infiltradas nas pálpebras superior e inferior direitas. / Figure 3: Patient 2:

Ulcerative and vegetative lesion with bed showing stippled bemorrhage and slightly infiltrated borders in the right superior and inferior eyelids. 
pensa após 15 dias por haver redução significativa do processo inflamatório. Obteve-se rápida regressão da lesão sem seqüelas (Figura 4).

\section{DISCUSSÃO}

O comprometimento ocular na paracoccidioidomicose é raro, tendo sido descrito pela primeira vez por Terra em 1923..$^{12}$ Desde então foram relatados apenas 55 casos na literatura mundial (fonte Medline e Lilacs), sendo a maioria deles no Brasil. Neste trabalho, apresentam-se uma síntese desses casos e, especialmente, dois relatos de paracoccidioidomicose cuja primeira manifestação ocorreu na região ocular (Tabela 1).

As áreas mais frequientemente acometidas na região ocular são as pálpebras e a conjuntiva, sendo raras outras localizações. ${ }^{10}$ Nos dois casos relatados há envolvimento das pálpebras e da mucosa conjuntival. A análise dos 55 casos descritos mostra que ocorreu envolvimento palpebral exclusivo em 23 deles; envolvimento palpebral e de outras estruturas oculares em 21; e envolvimento exclusivo de outras estruturas oculares que não a pálpebra em 11 casos. As outras estruturas em que já foi descrito acometimento ocular incluem córnea, conjuntiva, esclera, coróide, úvea e retina. Os achados permitem sugerir que, frente ao diagnóstico de paracoccidioidomicose com envolvimento palpebral, torna-se necessária a avaliação oftalmológica completa para certificar-se do não envolvimento de outras estruturas.

Em geral o acometimento do globo ocular e anexos acompanha-se do comprometimento de outros órgãos. ${ }^{10}$ Nos casos aqui descritos nenhum dos pacientes apresentou alterações radiológicas pulmonares, apesar de não terem sido realizados exames adicionais de maior sensibilidade, não corroborando o envolvimento multifocal que a doença geralmente apresenta. No entanto o paciente 1 apresentava lesões também no braço, o que pode sugerir uma possível disseminação hematogênica.

$\mathrm{Na}$ região ocular, as lesões iniciam comumente como pápula eritematosa na borda palpebral que evolui com crescimento progressivo e tendência à ulceração central. As úlceras apresentam bordas elevadas e fundo com granulação fina, e pontilhado hemorrágico característico, que na mucosa oral é conhecido como estomatite moriforme. ${ }^{47}$ As lesões podem evoluir com destruição dos

Figura 4: Paciente 2: dois meses após tratamento lowing significant reduction in the inflammatory process. $A$ rapid regression of the lesions was acheived without sequels (Figure 4).

\section{DISCUSSION}

Ocular impairment in paracoccidioidomycosis is rare, since being described for the first time by Terra in $1923,{ }^{12}$ only 55 cases have been reported in the world literature (source: Medline and Lilacs) and of these, most were in Brazil. The present work offers a synthesis of these cases and in particular two paracoccidioidomycosis reports, the first manifestation of which occurred in the ocular area (Table 1).

The areas most frequently involved in the ocular region are the eyelids and conjunctiva, while other locations are rarely affected. 10 In both case reports there was involvement of the eyelids and conjunctival mucous membrane. Analysis of the 55 cases reported to date showed that exclusive palpebral involvement occurred in 23; palpebral involvement and of other ocular structures in 21; and exclusive involvement of ocular structures other than the eyelid in 11 cases. The other structures in which ocular involvement has already been described include the cornea, conjunctive, sclera, choroidea, uvea and retina. The findings point to the fact that, faced with a diagnosis of paracoccidioidomycosis with palpebral involvement, a complete ophthalmologic evaluation is necessary to certify whether other structures are affected.

In general, involvement of the eyeball and enclosures is accompanied by the compromising of other organs. ${ }^{10}$ In the cases described here, neither of the patients presented pulmonary radiological alterations, albeit that additional exams of greater sensitivity were not performed, which does not corroborate with the multifocal involvement that the disease usually presents. However, Patient 1 also presented lesions in the arm, which can suggest a possible hematogenic dissemination.

In the ocular region, the lesions commonly begin as an erythematosus papule in the palpebral border that courses with progressive growth and tendency to central

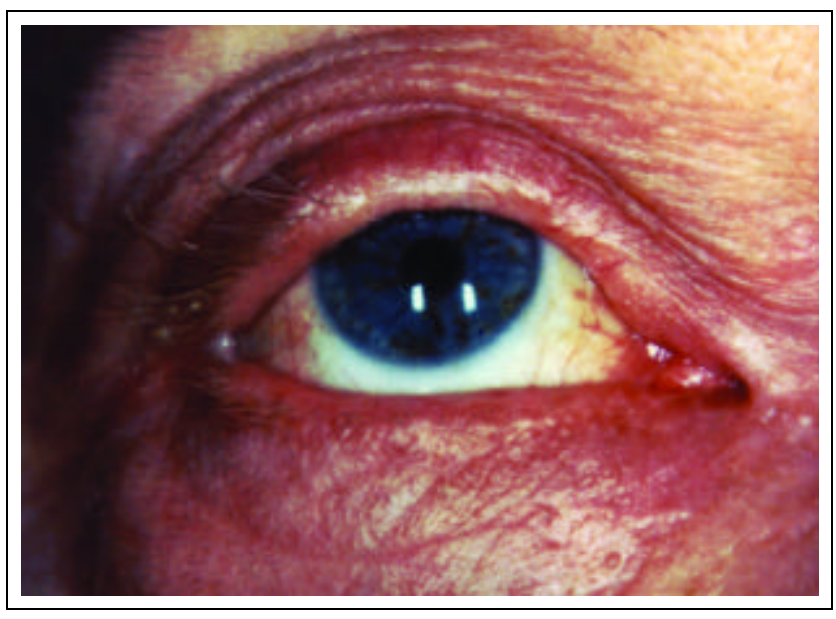
ulceration. The ulcers present raised borders and bed with fine granulation and characteristic stippled hemorrhage, which in the oral mucous membrane is known as "moriforme" stomatitis. ${ }^{47}$ The lesions can develop with

Figure 4: Patient 2. two months after treatment 
Tabela 1: Revisão dos casos de paracoccidioidomicose da região ocular descritos na literatura.

Table 1: Revision of ocular paracoccidioidomycosis cases described in the literature within the region.

\begin{tabular}{|c|c|c|c|c|c|c|c|c|}
\hline $\begin{array}{l}\text { Autores } \\
\text { Authors }\end{array}$ & $\begin{array}{l}\text { Ano e } \mathrm{n}^{\circ} \text { de Casos } \\
\text { Publicados / Year } \\
\text { and } n^{\circ} \text { of Cases } \\
\text { Published }\end{array}$ & $\begin{array}{l}\text { Idade } \\
\text { Age }\end{array}$ & $\begin{array}{l}\text { Sexo } \\
\text { Sex }\end{array}$ & $\begin{array}{l}\text { Cor } \\
\text { Color }\end{array}$ & $\begin{array}{l}\text { Profissão } \\
\text { Profession }\end{array}$ & $\begin{array}{l}\text { Origem } \\
\text { Origin }\end{array}$ & $\begin{array}{l}\text { Olho } \\
\text { Eye }\end{array}$ & $\begin{array}{l}\text { Localização das } \\
\text { Lesões / Localization } \\
\text { of Lesions }\end{array}$ \\
\hline Terra $^{12}$ & 1923 (1 caso/case) & 40 & $\mathrm{M} / M$ & $\mathrm{~B} / W$ & - & Brasil/Brazil & $\mathrm{AO} / B E$ & Pálpebra /Eyelid \\
\hline Ferguson $^{13}$ & 1928 ( 1 caso/case $)$ & 58 & $\mathrm{M} / M$ & $\mathrm{~B} / W$ & - & - & $\mathrm{E} / L$ & $\begin{array}{l}\text { Córnea e esclera } \\
\text { Cornea and sclera }\end{array}$ \\
\hline Belfort $^{14}$ & 1930 (1 caso/case) & 52 & $\mathrm{M} / M$ & $\mathrm{~B} / W$ & - & Brasil/Brazil & $\mathrm{D} / R$ & $\begin{array}{l}\text { Pálpebra e conjuntiva } \\
\text { Eyelid and conjunctiva }\end{array}$ \\
\hline Campos $^{15}$ & 1930 (1 caso/case) & 52 & $\mathrm{M} / M$ & $\mathrm{~B} / W$ & - & Brasil/Brazil & $\mathrm{AO} / B E$ & $\begin{array}{l}\text { Conjuntiva, pálpebras } \\
\text { superior e inferior } \\
\text { Conjunctiva, superior } \\
\text { and inferior eyelids }\end{array}$ \\
\hline Silva $^{16}$ & 1936 (3 casos/cases) & - & $\mathrm{M} / M$ & - & - & Brasil/Brazil & $\mathrm{D} / R$ & Pálpebra/Eyelid \\
\hline & & - & $\mathrm{M} / M$ & - & - & Brasil/Brazil & $\mathrm{E} / L$ & Pálpebra/Eyelid \\
\hline & & 44 & $\mathrm{M} / M$ & $\mathrm{~N} / B$ & $\begin{array}{l}\text { Jardineiro } \\
\text { Gardener }\end{array}$ & Brasil/Brazil & $\mathrm{AO} / B E$ & Pálpebra/Eyelid \\
\hline Andrade $^{17}$ & 1937 (1 caso/case) & \multicolumn{7}{|c|}{ Caso já citado por Silva / Case already cited by Silva } \\
\hline Almeida $^{18}$ & 1939 (1 caso/case) & 39 & $\mathrm{M} / M$ & $\mathrm{~B} / W$ & $\begin{array}{l}\text { Agricultor } \\
\text { Farm worker }\end{array}$ & Brasil/Brazil & $\mathrm{D} / R$ & Pálpebra/Eyelid \\
\hline Andrade ${ }^{19}$ & 1939 (1 caso/case) & - & - & - & - & Brasil/Brazil & $\mathrm{D} / R$ & Pálpebra/Eyelid \\
\hline Queiroz ${ }^{20}$ & 1943 (1 caso/case) & - & - & - & - & Brasil/Brazil & - & Pálpebra/Eyelid \\
\hline Sales $^{21}$ & 1945 (1 caso/case) & 37 & $\mathrm{M} / M$ & $\mathrm{~N} / B$ & $\begin{array}{l}\text { Mecânico } \\
\text { Mechanic }\end{array}$ & Brasil/Brazil & $\mathrm{D} / R$ & $\begin{array}{l}\text { Pálpebras superior e } \\
\text { inferior / Superior } \\
\text { and inferior eyelids }\end{array}$ \\
\hline Lacaz $^{22}$ & 1948 (1 caso/case) & - & $\mathrm{M} / M$ & - & - & Brasil/Brazil & - & Pálpebra/Eyelid \\
\hline $\mathrm{Niño}^{23}$ & 1950 (1 caso/case) & 42 & $\mathrm{M} / M$ & - & $\begin{array}{l}\text { Soldado } \\
\text { Soldier }\end{array}$ & Argentina & $\mathrm{E} / L$ & $\begin{array}{l}\text { Pálpebra inferior } \\
\text { Inferior eyelid }\end{array}$ \\
\hline Rocha $^{24}$ & 1952 (3 casos/cases) & 32 & $\mathrm{M} / M$ & $\mathrm{~B} / W$ & $\begin{array}{l}\text { Mercante } \\
\text { Merchant }\end{array}$ & Brasil/Brazil & $\mathrm{E} / L$ & $\begin{array}{l}\text { Pálpebra inferior } \\
\text { Inferior eyelid }\end{array}$ \\
\hline \multicolumn{9}{|c|}{ Os outros dois casos já foram publicados por Almeida e Sales / The other cases were already described by Almeida and Sales } \\
\hline Azevedo ${ }^{25}$ & 1954 (1 caso/case) & - & $\mathrm{M} / M$ & - & - & Brasil/Brazil & - & Pálpebra/Eyelid \\
\hline Gaipa $^{26}$ & 1957 (1 caso/case) & - & - & - & - & Venezuela & - & Conjuntiva/Conjunctiva \\
\hline $\begin{array}{l}\text { Gonzáles } \\
\text { Ochoa }^{27}\end{array}$ & 1957 (1 caso/case) & 37 & $\mathrm{M} / M$ & - & $\begin{array}{l}\text { Agricultor } \\
\text { Farm worker }\end{array}$ & México & $\mathrm{E} / L$ & Pálpebra/Eyelid \\
\hline
\end{tabular}




\begin{tabular}{|c|c|c|c|c|c|c|c|c|}
\hline $\begin{array}{l}\text { Autores } \\
\text { Authors }\end{array}$ & $\begin{array}{l}\text { Ano e } \mathrm{n}^{\circ} \text { de Casos } \\
\text { Publicados / Year } \\
\text { and } n^{\circ} \text { of Cases } \\
\text { Published }\end{array}$ & $\begin{array}{l}\text { Idade } \\
\text { Age }\end{array}$ & $\begin{array}{l}\text { Sexo } \\
\text { Sex }\end{array}$ & $\begin{array}{l}\text { Cor } \\
\text { Color }\end{array}$ & $\begin{array}{l}\text { Profissão } \\
\text { Profession }\end{array}$ & $\begin{array}{l}\text { Origem } \\
\text { Origin }\end{array}$ & $\begin{array}{l}\text { Olho } \\
\text { Eye }\end{array}$ & $\begin{array}{l}\text { Localização das } \\
\text { Lesões / Localization } \\
\text { of Lesions }\end{array}$ \\
\hline Haedo $^{28}$ & 1957 (1 caso/case) & 53 & $\mathrm{M} / M$ & - & $\begin{array}{l}\text { Agricultor } \\
\text { Farm worker }\end{array}$ & $\begin{array}{l}\text { Paraguai } \\
\text { Paraguay }\end{array}$ & $\mathrm{E} / L$ & $\begin{array}{l}\text { Pálpebra superior } \\
\text { Superior eyelid }\end{array}$ \\
\hline Blodi $^{29}$ & 1958 ( 1 caso/case $)$ & 57 & $\mathrm{M} / M$ & $\mathrm{~B} / W$ & - & - & $\mathrm{AO} / B E$ & $\begin{array}{l}\text { Pálpebras superior e } \\
\text { inferior / Superior and } \\
\text { inferior eyelids }\end{array}$ \\
\hline \multirow[t]{2}{*}{ Campos $^{30}$} & 1960 ( 2 casos/cases) & 76 & $\mathrm{M} / M$ & $\mathrm{~B} / W$ & $\begin{array}{l}\text { Jardineiro } \\
\text { Gardener }\end{array}$ & Brasil/Brazil & $\mathrm{D} / R$ & $\begin{array}{l}\text { Córnea, pálpebras } \\
\text { superior e inferior } \\
\text { Cornea, superior and } \\
\text { inferior eyelids }\end{array}$ \\
\hline & & 56 & $\mathrm{M} / M$ & $\mathrm{~B} / W$ & $\begin{array}{l}\text { Encanador } \\
\text { Plumber }\end{array}$ & Brasil/Brazil & $\mathrm{D} / R$ & $\begin{array}{l}\text { Conjuntiva e pálpebra } \\
\text { inferior / Conjunctiva } \\
\text { and inferior eyelid }\end{array}$ \\
\hline \multirow{3}{*}{$\begin{array}{l}\text { Machado } \\
\text { Filho }^{31}\end{array}$} & 1960 (3 casos/cases) & - & - & - & - & Brasil/Brazil & - & Conjuntiva/Conjunctiva \\
\hline & & - & - & - & - & Brasil/Brazil & - & Conjuntiva/Conjunctiva \\
\hline & & - & - & - & - & Brasil/Brazil & - & Conjuntiva/Conjunctiva \\
\hline Conti-Diaz ${ }^{32}$ & 1960 (1 caso/case) & 38 & $\mathrm{M} / M$ & $\mathrm{~B} / W$ & $\begin{array}{l}\text { Segurança } \\
\text { Security agent }\end{array}$ & $\begin{array}{l}\text { Uruguai } \\
\text { Uruguay }\end{array}$ & $\mathrm{E} / L$ & Coróides / Choroidea \\
\hline Servino $^{33}$ & 1966 ( 1 caso/case $)$ & 48 & $\mathrm{M} / M$ & $\mathrm{~B} / W$ & Agricultor & Argentina & $\mathrm{D} / R$ & $\begin{array}{l}\text { Pálpebras, conjuntiva } \\
\text { e córnea /Farm worker } \\
\text { Eyelids, conjunctiva } \\
\text { and cornea }\end{array}$ \\
\hline \multirow[t]{2}{*}{ Negroni $^{34}$} & 1968 ( 2 casos/cases) & 59 & $\mathrm{M} / M$ & - & $\begin{array}{l}\text { Agricultor } \\
\text { Farm worker }\end{array}$ & Argentina & $\mathrm{D} / R$ & $\begin{array}{l}\text { Pálpebra inferior } \\
\text { Inferior eyelid }\end{array}$ \\
\hline & & 11 & $\mathrm{~F} / F$ & - & - & Argentina & $\mathrm{D} / R$ & Órbita / Orbit \\
\hline Brass $^{35}$ & 1969 (1 caso/case) & - & - & - & - & Venezuela & - & Conjuntiva/Conjunctiva \\
\hline Brick $^{36}$ & 1969 (1 caso/case) & 28 & $\mathrm{M} / M$ & $\mathrm{~N} / B$ & - & Brasil/Brazil & $\mathrm{D} / R$ & $\begin{array}{l}\text { Pálpebra inferior } \\
\text { Inferior eyelid }\end{array}$ \\
\hline \multirow[t]{2}{*}{ Dantas $^{37,38}$} & 1971 ( 2 casos/cases) & 55 & $\mathrm{M} / M$ & $\mathrm{~N} / B$ & $\begin{array}{l}\text { Estivador } \\
\text { Stevedore }\end{array}$ & Brasil/Brazil & $\mathrm{E} / L$ & $\begin{array}{l}\text { Úvea anterior, } \\
\text { pálpebras superior e } \\
\text { inferior / Anterior } \\
\text { uvea, superior and } \\
\text { inferior eyelids }\end{array}$ \\
\hline & & 39 & $\mathrm{M} / M$ & $\mathrm{~N} / B$ & Militar/Soldier & Brasil/Brazil & $\mathrm{E} / L$ & $\begin{array}{l}\text { Retina e úvea. } \\
\text { Retina and uvea }\end{array}$ \\
\hline Albornoz $^{39}$ & 1972 (1 caso/case) & - & - & - & - & Venezuela & $\mathrm{D} / R$ & $\begin{array}{l}\text { Pálpebra inferior } \\
\text { Inferior eyelid }\end{array}$ \\
\hline
\end{tabular}




\begin{tabular}{|c|c|c|c|c|c|c|c|c|}
\hline $\begin{array}{l}\text { Autores } \\
\text { Authors }\end{array}$ & $\begin{array}{l}\text { Ano e } n^{\circ} \text { de Casos } \\
\text { Publicados / Year } \\
\text { and } n^{\circ} \text { of Cases } \\
\text { Published }\end{array}$ & $\begin{array}{l}\text { Idade } \\
\text { Age }\end{array}$ & $\begin{array}{l}\text { Sexo } \\
\text { Sex }\end{array}$ & $\begin{array}{l}\text { Cor } \\
\text { Color }\end{array}$ & $\begin{array}{l}\text { Profissão } \\
\text { Profession }\end{array}$ & $\begin{array}{l}\text { Origem } \\
\text { Origin }\end{array}$ & $\begin{array}{l}\text { Olho } \\
\text { Eye }\end{array}$ & $\begin{array}{l}\text { Localização das } \\
\text { Lesões / Localization } \\
\text { of Lesions }\end{array}$ \\
\hline Belfort $\mathrm{Jr}^{40}$ & 1975 (1 caso/case) & 35 & $\mathrm{M} / M$ & $\mathrm{~B} / W$ & $\begin{array}{l}\text { Agricultor } \\
\text { Farm worker }\end{array}$ & Brasil/Brazil & $\mathrm{E} / L$ & $\begin{array}{l}\text { Pálpebra e conjuntiva } \\
\text { Eyelid and conjunctiva }\end{array}$ \\
\hline Del Negro 9 & 1982 (1 caso/case) & 42 & $\mathrm{M} / M$ & - & $\begin{array}{l}\text { Agricultor } \\
\text { Farm worker }\end{array}$ & Brasil/Brazil & $\mathrm{D} / R$ & $\begin{array}{l}\text { Pálpebra inferior e } \\
\text { conjuntiva / Inferior } \\
\text { eyelid and conjunctiva }\end{array}$ \\
\hline Bonomo $^{42}$ & 1982 ( 1 caso/case $)$ & 48 & $\mathrm{~F} / F$ & - & $\begin{array}{l}\text { Agricultor } \\
\text { Farm worker }\end{array}$ & Brasil/Brazil & $\mathrm{E} / L$ & $\begin{array}{l}\text { Coróide e pálpebra } \\
\text { superior / Choroidea } \\
\text { and superior eyelid }\end{array}$ \\
\hline Cechella ${ }^{43}$ & 1982 (1 caso/case) & 44 & $\mathrm{M} / M$ & - & $\begin{array}{l}\text { Agricultor } \\
\text { Farm worker }\end{array}$ & Brasil/Brazil & $\mathrm{E} / L$ & $\begin{array}{l}\text { Pálpebra superior } \\
\text { Superior eyelid }\end{array}$ \\
\hline Jannke ${ }^{44}$ & 1983 (1 caso) & 39 & $\mathrm{M} / M$ & - & $\begin{array}{l}\text { Agricultor } \\
\text { Farm worker }\end{array}$ & Brasil/Brazil & $\mathrm{E} / L$ & $\begin{array}{l}\text { Pálpebras superior e } \\
\text { inferior / Superior } \\
\text { and inferior eyelids }\end{array}$ \\
\hline Arruda $^{45}$ & 1986 (1 caso/case) & 56 & $\mathrm{M} / M$ & - & $\begin{array}{l}\text { Agricultor } \\
\text { Farm worker }\end{array}$ & Brasil/Brazil & $\mathrm{E} / L$ & Coróides / Choroidea \\
\hline Pinheiro $^{46}$ & 1987 (1 caso/case) & 22 & $\mathrm{~F} / F$ & $\mathrm{~N} / B$ & $\begin{array}{l}\text { Doméstica } \\
\text { Maid }\end{array}$ & Brasil/Brazil & $\mathrm{D} / R$ & $\begin{array}{l}\text { Pálpebra, coróides e } \\
\text { retina / Eyelid, } \\
\text { Choroidea and retina }\end{array}$ \\
\hline \multirow[t]{6}{*}{$\begin{array}{l}\text { Moraes } \\
\text { Silva }^{47}\end{array}$} & \multirow[t]{6}{*}{1988 (6 casos/cases) } & 33 & $\mathrm{M} / M$ & $\mathrm{~B} / W$ & $\begin{array}{l}\text { Tratorista } \\
\text { Tractor driver }\end{array}$ & Brasil/Brazil & $\mathrm{E} / L$ & $\begin{array}{l}\text { Pálpebra, córnea e } \\
\text { úvea./ Eyelid, cornea } \\
\text { and uvea }\end{array}$ \\
\hline & & 48 & $\mathrm{M} / M$ & $\mathrm{~B} / W$ & $\begin{array}{l}\text { Agricultor } \\
\text { Farm worker }\end{array}$ & Brasil/Brazil & $\mathrm{E} / L$ & $\begin{array}{l}\text { Pálpebra e conjuntiva } \\
\text { Eyelid and conjunctiva }\end{array}$ \\
\hline & & 57 & $\mathrm{M} / M$ & $\mathrm{~B} / W$ & $\begin{array}{l}\text { Agricultor } \\
\text { Farm worker }\end{array}$ & Brasil/Brazil & $\mathrm{E} / L$ & $\begin{array}{l}\text { Pálpebra e conjuntiva } \\
\text { Eyelid and conjunctiva }\end{array}$ \\
\hline & & 49 & $\mathrm{M} / M$ & $\mathrm{~B} / W$ & $\begin{array}{l}\text { Agricultor } \\
\text { Farm worker }\end{array}$ & Brasil/Brazil & $\mathrm{E} / L$ & Pálpebra / Eyelid \\
\hline & & 54 & $\mathrm{M} / M$ & $\mathrm{~N} / B$ & $\begin{array}{l}\text { Agricultor } \\
\text { Farm worker }\end{array}$ & Brasil/Brazil & $\mathrm{E} / L$ & $\begin{array}{l}\text { Pálpebra e conjuntva } \\
\text { Eyelid and conjunctiva }\end{array}$ \\
\hline & & 48 & $\mathrm{M} / M$ & $\mathrm{~B} / W$ & $\begin{array}{l}\text { Agricultor } \\
\text { Farm worker }\end{array}$ & Brasil/Brazil & $\mathrm{E} / L$ & $\begin{array}{l}\text { Pálpebra e conjuntva } \\
\text { Eyelid and conjunctiva }\end{array}$ \\
\hline Salinas $^{48}$ & 1989 ( 1 caso/case $)$ & 36 & $\mathrm{M} / M$ & $\mathrm{~N} / B$ & $\begin{array}{l}\text { Agricultor } \\
\text { Farm worker }\end{array}$ & Brasil/Brazil & $\mathrm{E} / L$ & $\begin{array}{l}\text { Pálpebra e conjuntiva } \\
\text { Eyelid and conjunctiva }\end{array}$ \\
\hline Lottemberg ${ }^{49}$ & 1992 (1 caso/case) & 32 & $\mathrm{M} / M$ & $\mathrm{~N} / B$ & - & Brasil/Brazil & $\mathrm{E} / L$ & Retina / Retina \\
\hline Carneiro $^{50}$ & 1995 (4 casos/cases) & 42 & $\mathrm{M} / M$ & - & $\begin{array}{l}\text { Agricultor } \\
\text { Farm worker }\end{array}$ & Brasil/Brazil & $\mathrm{AO} / B E$ & $\begin{array}{l}\text { Pálpebra, conjuntiva } \\
\text { e córnea / Eyelid, } \\
\text { conjunctiva and cornea }\end{array}$ \\
\hline
\end{tabular}




\begin{tabular}{|c|c|c|c|c|c|c|c|c|}
\hline $\begin{array}{l}\text { Autores } \\
\text { Authors }\end{array}$ & $\begin{array}{l}\text { Ano e } \mathrm{n}^{\circ} \text { de Casos } \\
\text { Publicados / Year } \\
\text { and } n^{\circ} \text { of Cases } \\
\text { Published }\end{array}$ & $\begin{array}{l}\text { Idade } \\
\text { Age }\end{array}$ & $\begin{array}{l}\text { Sexo } \\
\text { Sex }\end{array}$ & $\begin{array}{l}\text { Cor } \\
\text { Color }\end{array}$ & $\begin{array}{l}\text { Profissão } \\
\text { Profession }\end{array}$ & $\begin{array}{l}\text { Origem } \\
\text { Origin }\end{array}$ & $\begin{array}{l}\text { Olho } \\
\text { Eye }\end{array}$ & $\begin{array}{l}\text { Localização das } \\
\text { Lesões / Localization } \\
\text { of Lesions }\end{array}$ \\
\hline & & 52 & $\mathrm{~F} / F$ & - & $\begin{array}{l}\text { Agricultora } \\
\text { Farm worker }\end{array}$ & Brasil/Brazil & $\mathrm{E} / L$ & $\begin{array}{l}\text { Pálpebra e conjuntiva } \\
\text { Eyelid and conjunctiva }\end{array}$ \\
\hline & & 68 & $\mathrm{M} / M$ & - & $\begin{array}{l}\text { Agricultor } \\
\text { Farm worker }\end{array}$ & Brasil/Brazil & $\mathrm{E} / L$ & $\begin{array}{l}\text { Pálpebra e conjuntiva } \\
\text { bulbar /Eyelid and } \\
\text { conjunctiva bulbar }\end{array}$ \\
\hline & & 52 & $\mathrm{M} / M$ & - & $\begin{array}{l}\text { Comerciante } \\
\text { Business man }\end{array}$ & Brasil/Brazil & $\mathrm{E} / L$ & Pálpebra/Eyelid \\
\hline Noronha $^{51}$ & 1998 (1 caso/case) & 54 & $\mathrm{M} / M$ & - & $\begin{array}{l}\text { Motorista } \\
\text { Motorist }\end{array}$ & Brasil/Brazil & $\mathrm{D} / R$ & $\begin{array}{l}\text { Pálpebra, córnea, } \\
\text { vítreo e retina } \\
\text { Eyelid, cornea, } \\
\text { vitreum and retina }\end{array}$ \\
\hline Tobon $^{52}$ & 1998 (1 caso/case) & 30 & $\mathrm{M} / M$ & - & $\begin{array}{l}\text { Agricultor } \\
\text { Farm worker }\end{array}$ & $\begin{array}{l}\text { Colômbia } \\
\text { Columbia }\end{array}$ & $\mathrm{E} / L$ & Pálpebra/Eyelid \\
\hline Ferraz $^{53}$ & 2001 (1 caso/case) & 49 & $\mathrm{M} / M$ & - & Pedreiro & Brasil/Brazil & $\mathrm{E} / L$ & $\begin{array}{l}\text { Pálpebra e conjuntiva } \\
\text { Eyelid and conjunctiva }\end{array}$ \\
\hline \multicolumn{9}{|c|}{$\begin{array}{l}\text { M: Masculino / M: Male } \\
\text { F: Feminino / F: Female } \\
\text { B: Branco / W: White } \\
\text { N: Negro / B: Black } \\
\text { AO: Ambos olhos / BE: Both eyes } \\
\text { E: Esquerdo / L: Left } \\
\text { D: Direito / R: Right }\end{array}$} \\
\hline
\end{tabular}

ductos lacrimais e de todas as camadas da pálpebra ou com formação de coloboma palpebral, incluindo comprometimento da conjuntiva bulbar e da córnea, por contigüidade. ${ }^{47}$ Os linfonodos pré-auriculares podem ser palpáveis. ${ }^{40}$

O diagnóstico diferencial deve ser feito na fase inicial com hordéolo e blefarite bacteriana, e, na fase crônica, quando as lesões são mais extensas, com carcinomas basocelular e espinocelular, tracoma, leishmaniose, histoplasmose, esporotricose, lúpus eritematoso crônico cutâneo discóide, tuberculose e sífilis secundária. ${ }^{40,41}$ Tendo em vista a alta freqüência da paracoccidioidomicose no Brasil, deve-se sempre considerar essa doença como diagnóstico diferencial de lesões ulceradas na região palpebral, principalmente nos pacientes do sexo masculino com idade superior a 30 anos e história de contato com o meio rural. ${ }^{2}$

A comprovação diagnóstica pode ser realizada mediante exame micológico direto do exsudato das lesões ou fragmento de tecido, em que se observa o fungo com seu aspecto característico em "roda de leme".,3,4 O exame microscópico da cultura em ágar-chocolate e ágar-sangue a $37^{\circ} \mathrm{C}$ apresenta, após período de sete a 10 dias, as formas clássicas do Paracoccidioides brasiliensis. Em ágarSaboraud em temperatura ambiente entre 20 e 30 dias, podem ser observados hifas e clamidiosporos. ${ }^{3,5,11} \mathrm{O}$ exame histopatológico também é bastante útil, especialmente destruction of the tear ducts and of all the layers of the eyelid or with the formation of palpebral coloboma, including involvement of the bulbar conjunctiva and the cornea by contiguity. ${ }^{47}$ The pre-auricular lymph nodes can be palpable. ${ }^{40}$

The differential diagnosis should be made in the initial phase with hordeolum and bacterial blepharitis, while in the chronic phase, when the lesions are more extensive, with basal cell and squamous cell carcinomas, trachoma, leishmaniosis, histoplasmosis, sporotrichosis, chronic discoid cutaneous lupus erythematosus, tuberculosis and secondary syphilis. ${ }^{40,41}$ Bearing in mind the high frequency of paracoccidioidomycosis in Brazil, one should always consider this disease in the differential diagnosis of ulcerated lesions in the palpebral area, especially among male patients over 30 years old and history of contact with rural environments. ${ }^{2}$

Diagnostic confirmation can be accomplished by direct mycological exam of the exudate of the lesions or tissue sample, in which the fungi can be identified by its characteristic "ship's wheel" aspect. ${ }^{2,3,4}$ Microscopic exam of the culture in chocolate-agar and blood-agar at $37^{\circ} \mathrm{C}$, after a period of seven to 10 days, presents the classic forms of Paracoccidioides brasiliensis. In Sabouraud's agar cultivated at room temperature for 20 to 30 days, hyphae and 
quando há poucos microorganismos. ${ }^{2,3,4}$ Observa-se reação granulomatosa rica em células epitelióides e gigantócitos, no interior das quais pode ser encontrado o Paracoccidioides brasiliensis. ${ }^{54}$

O tratamento é realizado com medicações sistêmicas, havendo várias possibilidades, entre as quais os derivados sulfamídicos, a anfotericina B e os derivados imidazólicos. Esses são os mais freqüentemente utilizados, sendo o itraconazol considerado a droga de escolha por alguns autores, devido ao tempo de tratamento mais breve em relação a outras drogas administradas por via oral (de três a seis meses), menor ocorrência de efeitos colaterais e menor taxa de recidiva (entre três e 5\%). ${ }^{3,455,56}$ Recentemente foi descrito o uso bem-sucedido de terbinafina em paciente com lesões cutâneas e mucosas na região genital, na dose de $250 \mathrm{mg}$ duas vezes por dia durante seis meses. ${ }^{57}$

Conclui-se que o envolvimento ocular na paracoccidioidomicose é incomum e, quando presente, acomete mais frequientemente as pálpebras e a conjuntiva. Apesar de ser localização rara, deve ser considerada nos diagnósticos diferenciais de lesões ulceradas da pálpebra, pois é doença endêmica no Brasil. Ressalta-se também a importância de avaliação sistêmica, visto ser doença geralmente multifocal. Recomenda-se o exame oftalmológico completo, por haver casos descritos de envolvimento de outras estruturas oculares, podendo a doença evoluir para formas mais graves com comprometimento da visão.

\section{REFERÊNCIAS / REFERENCES}

1. Padilha-Gonçalves A. Paracoccidioidomicose. An Bras Dermatol 1985; 60(1):271-80.

2. Rios-Fabra A, Moreno AR, Istúriz RE. Fungal Infection in Latin American Countries. Infect Dis Clin North Am 1994; 8(1):129-54. 3. Brummer E, Castaneda E, Restrepo A. Paracoccidioidomycosis: an Update. Clin Microbiol Rev 1993; 6(2):89-117.

4. Negroni R. Paracoccidioidomycosis (South American Blastomycosis, Lutz's Mycosis). Int J Dermatol 1993; 32(12):847-59. 5. Severo LC. Paracoccidioidomicose. In: Silva LCC. Compêndio de Pneumologia.2a edição. São Paulo: BYK; 1993. p.601-6

6. Restrepo A. The ecology of Paracoccidioides brasiliensis: a puzzle still unsolved. J Med Vet Mycol 1985; 23:323-34.

7. Restrepo A, Greer DL. La epidemiologia de la paracoccidioidomicosis. Bol Of Sanit Panam 1977; 83:428-45.

8. Montenegro MRG. Formas Clínicas da Paracoccidioidommicose. Rev Inst Med Trop S Paulo 1986; 28(3):203-4.

9. Fisher F, Cook NB. Fungos Sistêmicos. In: Fisher F, Cook NB. Micologia Fundamentos e Diagnostico. $1^{\circ}$ Edição. Rio de Janeiro: Revinter; 2001 p.227-250.

10. Del Negro G. Outras lesões. Formas de ocorrência rara e associações a outros processos. In: Del Negro G, Lacaz CS, Fiorillo AM, eds. Paracoccidioidomicose (Blastomicose sul-americana). São Paulo: Saraiva, EDUSP, 1982: 229-243.

11. Lacaz CS, Porto E, Martinho JEC. Paracoccidioidommicose. In: Lacaz CS, Porto E, Martinho JEC. Micologia Médica: fungos, actinomicetos e algas de interesse médico. 7a edição. São Paulo: Sarvier; 1984. p.189-216.

12. Terra F. Três casos de Blastomycose. Brasil-med 1923; 32: 41-4. chlamydiaspores ${ }^{3,5,11}$ can be observed. Histopathological exam is also quite useful, especially when there are few microorganisms. ${ }^{2,3,4}$ A granulomatous reaction rich in epithelioid cells and gigantocytes is observed, inside which Paracoccidioides brasiliensis can be found..$^{54}$

Treatment can be accomplished using systemic medications, with several possibilities, including sulfamides, imidazoles and amphotericin B. These are the most frequently used and itraconazole is considered the drug of choice by some authors, due to the shorter treatment duration in relation to other orally administered drugs (from three to six months) as well as less frequent occurrence of side effects and recurrence (between 3 and 5\%).,3,55,56 Recently the successful use of terbinafine was described in a patient with cutaneous and mucous lesions in the genital area, at a dose of $250 \mathrm{mg}$ twice daily for six months. ${ }^{57}$

It was concluded that ocular involvement in paracoccidioidomycosis is uncommon, but when present most frequently affects the eyelids and conjunctiva. In spite of being a rare location, it should be considered in the differential diagnoses of ulcerated lesions of the eyelid, since it is an endemic disease in Brazil. The importance is also emphasized for a systemic evaluation, as the disease is usually multifocal. Likewise, a complete ophthalmologic exam is recommended, in view of the cases described with involvement of other ocular structures and that the disease can progress to more serious forms with visual impairment.

13. Ferguson AS, Blastomycosis of eye and face secondary to lung infection. Brit Med J 1928; 1:442-443.

14. Belfort F. Um caso de 'blastomycose' conjuntical. S Paulo méd 1929/1930; 2:777-786.

15. Campos E, Silva Filho JF. Localização palpebro-conjuntival da blastomycose. Ann Oculist 1930; xx:221-222.

16. Silva F. Comentários em torno de alguns casos de Blastomycose por Paracoccidiodes brasiliensis observados na Bahia. Brasil-med 1936; 50:706-715.

17. Andrade C. Manifestações oculares nas doenças tropicais. Arq Clin Oftal Oto-rino-laringol 1937; 4:50-52.

18. Almeida A. Blastomicose palpebral. Arq Inst P Burnier (Campinas) 1939; 5:324.

19. Andrade C. Micoses. Oftalmologia Tropical (Sul-americana). Rio de Janeiro, Rodrigues e cia, 1940; 131-150.

20. Queiroz S. Blastomicose palpebral. Bol Soc Méd Cirurg, (Campinas) 1943; 3:130.

21. Sales M, Queiroz S. Blastomicose ocular (palpebral). Arq Inst P Burnier (Campinas) 1945; 7:260-261.

22. Lacaz CS. Blastomicose sul-americana. Na Inst Pinheiros 1948; 11:23-53.

23. Niño FL. Siete nuevas observaciones de granuloma paracoccidiodico em la Republica Argentina. Bol Inst Clin quir (B.Aires) 1950; 26:272-305.

24. Rocha M. Micosis em Oftalmologia. Arq Inst P Burnier (Campinas) 1952;9:28-69.

25. Azevedo PC. Algumas considerações sobre a blastomicose sul-americana e seu agente etiológico. Belém 1954: 95 p.(Thesis- 
Cathedral-School of Odontology of Pará).

26. Gaipa M. Su di caso di granulomatose paracoccidioidea con localizzazione congiuntivale. Rev Ital Trac 1957;9:6-13.

27. Gonzales Ochoa A, Domínguez Soto L. Blastomicosis sulamericana. Casos mexicanos. Rev Inst Salubr Enferm Trop (Méx), 1957; 17:97-104.

28. Haedo AA, Boggino J, Mercado M. Um caso de paracoccidiodomicosis conjuntival. Arq brás Oftal 1957; 20:450-457.

29. Blodi FC, Huffman WC. Cicatricial ectropion caused by cutaneous blastomycosis. Arch Ophthal 1958; 59:459-462.

30. Campos EC. Micose de Lutz (blastomicose sul-americana). Contribuição ao seu estudo no estado do Rio Grande do Sul. Porto Alegre, 1960: 220p. (Thesis-School of Medicine University of Rio Grande do Sul, Brasil).

31. Machado Filho J, Miranda JL. Considerações relativas à blastomicose sul-americana. Localizações, sintomas iniciais, vias de penetração e disseminação em 313 casos consecutivos. Hospital (Rio de J.) 1960; 58:129-137.

32. Conti-Diaz IA. Lesiones oculares em la blastomicosis sudamericana. Hospital (Rio de J.) 1960; 58:903-914.

33. Servino V, Proto F. Contributo alla conoscenza delle lesioni oculari da Paracoccidiodes brasiliensis. Boll Ocul 1966; 45:811-819.

34. Negroni R. Observaciones personales sobre la micosis de Lutz (blastomicosis sudamericana) en la Argentina. Buenos Aires, 1968: 78p.(Thesis-Doctorate, School of Medicine, University of Buenos Aires).

35. Brass K. Observaciones sobre la anatomia patológica, patogénesis y evolucion de la paracoccidioidomicosis. Mycopathologia 1969; 37: 119-138.

36. Brick M. Ocular involvement in lymphatic paracoccidioidomycosis. Acta Ophtal (Kbh.) 1969; 47:991-997.

37. Dantas AM, Curi R, Costa JD, Azulay RD, Quevedo LP, Manhães LF. Sobre um caso de Blastomicose sul-americana com lesão ocular. Rev Bras Oftal 1971; 30:83-91.

38. Dantas AM, Curi R, Silva JBC, Paiva LM. Blastomicose sulamericana. Relato de um caso com uveíte granulomatosa e oftalmoplegia externa incompleta. Rev Bras Oftal 1973; 32:61-68.

39. Albornoz MB. Paracoccidiodomicosis. Pan Amer Hlth Org Sci Publ 1972; 254:142-145.

40. Belfort JR, Fischman O, Camargo ZP, Almada A. Paracoccidioidomycosis with palpebral and conjuntival involvement. Mycopathologia 1975; 56(1): 21-4.

41. Sampaio SAP, Rivitti EA. Micoses Profundas. In: Sampaio SAP, Rivitti EA. Dermatologia. 2a edição. São Paulo: Editora Artes Médicas; 1998. p.535-561.

42. Bonomo PP, Belfort Jr R, Tsunechiro JY, G Filho O. Choroidal granuloma caused by Paracoccidiodes brasiliensis. A clinical and angiographic study. Mycopathologia 1982; 77:37-41.

42. Cechella MS, Melo CR, Melo IS, Londero AT, Barreto SM, Gaiger AM. Paracoccidiodomycose genital masculine. Rev Inst Med trop São Paulo 1982; 24:240-245.

44. Jannke HA, Lopez FS, Abrahão MCY, Thofern P, Duarte AL,
Holthausen ET. Blastomicose sul-americana palpebral. Rev bras Oftal 1983; 42:87-90.

45. Arruda WO, Canto MAS, Loddo G, Rebuffi VF, Cardoso MA. Paracoccidioidomicose ocular. Relato de um caso com corioretinite posterior. Rev Inst Med Trop São Paulo 1986; 28:190-193.

46. Pinheiro SRAA, Oréfice F, Mason EM. Blastomicose sulamericana: descrição de um caso com lesões cutâneas, nasais e envolvimento do trato uveal posterior. Arq Bras Oftal 1987; 50:66-69.

47. Silva MRBM, Mendes RP, Lastória JC, Barraviera B, Marques SA, Kamegasawa A. Paracoccidioidomycosis: Study of six cases with ocular involvement. Mycopathologia 1998; 102: 87-96.

48. Salinas LF, Pereira DM, Costa ER, Seixas J, Alves MR. Paracoccidioidomicose Ocular. Bras Méd 1989; 26(1-4):7-8.

49. Lottemberg C, Neves RA, Belfort Jr. R, Lowen MS, Colombo A, Rehder JR. Paracoccidioidomycosis chorioretinitis in a patient with Aids, Arq Bras Oftal 1992;55:13-14.

50. Carneiro EJS, Couto Júnior AS, França VP, Miranda D. Paracoccidioidomicose com envolvimento palpebral e conjuntival. Relato de 4 casos e revisão da literatura. Rev Bras Oftal 1995;54(10): 786-90.

51. Noronha RMJ, Perez NT, Fortes HM, Hueb M, Fontes CJF. Paracoccidioidomicose ocular: relato de um caso de doença multifocal com envolvimento da pálpebra. Arq Bras Oftal 1998; 61(5):590-592.

52. Tobon AM, Orozco B, Estrada S, Jaramillo E, De Bedout C, Arango M. Paracoccidioidomycosis and AIDS: Report of the first two Colombian cases. Rev Inst Med Trop São Paulo 1998; 40(6): 377-81.

53. Ferraz E, Cella W, Rocha E, Caldato R. Paracoccidioidomicose primária da pálpebra e conjuntiva. Arq Bras Oftal 2001; 64(3): 259-61.

54. Talhari S, Neves RG. Dermatologia tropical. $1^{\circ}$ ed São Paulo: MEDSI, 1985: 147-65.

55. San-Blas G. Paracoccidioidomycosis and its etiologic agent Paracoccidiodes brasiliensis. J Med Vet Mycol 1993; 31:99-113.

56. Del Negro G. Avanços terapêuticos em micoses profundas, com ênfase à paracoccidioidomicose. An bras Dermatol 1987; 62(4):209-12.

57. Ollague JM, Zuritta AM, Calero G. Paracoccidioidomycosis (South American blastomycosis) successfully treated with terbinafine: first case report. Br J Dermatol 2000; 143: 188-91.

\author{
ENDEREÇO PARA CORRESPONDÊNCIA: / MAILING ADDRESS: \\ Sergio Martinez Lecompte \\ Bocagrande Cra. 5ta \# 5-98 \\ Cartagena Colombia \\ E-mail: sergiomartinez@dermamail.com.br
}

